\title{
Optical bistability under non-resonant excitation in spinor polariton condensates
}

\author{
L. Pickup,${ }^{1}$ K. Kalinin,${ }^{2}$ A. Askitopoulos, ${ }^{1}$ Z. Hatzopoulos,${ }^{3,4}$ \\ P. G. Savvidis, ${ }^{3,5,6}$ N. G. Berloff, ${ }^{2,7}$ and P. G. Lagoudakis ${ }^{1,2}$ \\ ${ }^{1}$ School of Physics and Astronomy, University of Southampton, Southampton, SO17 1BJ, United Kingdom \\ ${ }^{2}$ Skolkovo Institute of Science and Technology, Skolkovo Innovation Center, Building 3, Moscow 143026, Russian Federation \\ ${ }^{3}$ Microelectronics Research Group, IESL-FORTH, P.O. Box 152\%, 71110 Heraklion, Crete, Greece \\ ${ }^{4}$ Department of Physics, University of Crete, 71003 Heraklion, Crete, Greece \\ ${ }^{5}$ Department of Materials Science and Technology, University of Crete, Crete, Greece \\ ${ }^{6}$ ITMO University, St. Petersburg 197101, Russia \\ ${ }^{7}$ Department of Applied Mathematics and Theoretical Physics, \\ University of Cambridge, Cambridge CB3 OWA, United Kingdom
}

(Dated: February 2, 2018)

\begin{abstract}
We realise bistability in the spinor of polariton condensates under non-resonant optical excitation and in the absence of biasing external fields. Numerical modelling of the system using the GinzburgLandau equation with an internal Josephson coupling between the two spin components of the condensate qualitatively describes the experimental observations. We demonstrate that polariton spin bistability strongly depends on the condensate's overlap with the exciton reservoir by tuning the excitation geometry and sample temperature. We obtain non-collapsing bistability hysteresis loops for a record range of sweep times, $[10 \mu s, 1 s]$, offering a promising route to spin switches and spin memory elements.
\end{abstract}

Optical bistability is the phenomenon of an optical system supporting two or more stable states for a given range of driving conditions [1,2]. A bistable behavior demonstrates an internal memory of the system, which could potentially be harnessed to form optical transistors and memory elements. It has been observed in systems such as cold atoms [3], lasers [4], self-electro-optic effect devices [5] and Fabry-Pérot cavities containing nonlinear materials $[6,7]$. Optical bistability in microcavity polaritons, the bosonic quasi-particles formed by the strong coupling of cavity photons and excitons, was previously demonstrated for resonant/quasi-resonant optical excitation [8-13], electrical biasing [14, 15] and non-resonant electrical injection [16]. In resonantly pumped microcavity polaritons, bistability was described by a Kerr-like nonlinearity resulting from polariton-polariton interactions [9] and by employing an analogy with optical parametric oscillators [10]. Under electrical injection, bistability was observed in the photoluminescence intensity, in the presence of an external magnetic field, and was attributed to the electrostatic screening of the injected charge carriers forming a positive feedback for the backward sweep of the driving current [16].

Bistability has also been realised in the spinor of a polariton state utilising polaritons' well defined spin and its one-to-one correspondence with the circular polarization of emitted light. Optical control of polariton spin was employed in the realisation of spin-bistability and multi-stability, using quasi-resonant optical excitation of a cylindrical mesa [8] by rotating the polarization of the optical pump. Recently, spin-bistability was also shown under non-resonant optical excitation in a mesa structured microcavity using a biasing electrical field to induce an energy splitting between two linear polarization modes, while keeping the optical excitation constant [14]. Under non-resonant pumping and in the absence of external fields, the preferable route for the operation of all-optical memory elements in polariton circuits, both polariton bistability and spin-bistability remain elusive, despite theoretical predictions [17].

For the observation of polariton bistability or spinbistability, it is essential that the population fluctuations of polaritons or the relative spin populations, remain sufficiently small so as not to bridge the two stable solutions of the system that would lead to a collapse of the hysteresis loop. Polaritons are an inherently open-dissipative, non-equilibrium system that requires continuous pumping to reach steady-state due to finite cavity lifetime. In the absence of external electric or magnetic fields, polariton bistability and spin-bistability has been observed only under resonant excitation; the fundamental difference between resonant and non-resonant optical pumping is the presence, in the latter case, of a hot-exciton reservoir, and the concomitant exciton-polariton interactions. Exciton-polariton pair scattering events contribute to the decoherence of polaritons $[18,19]$, however, the observed bistabilities in polariton systems occur at time scales that can exceed the coherence time of a polariton condensate by a factor of $10^{9}$, suggesting that it is not the exciton induced decoherence that precludes the observation of bistability under non-resonant pumping. Another source of instability that prevents bistable behavior under nonresonant pumping is the density fluctuations induced by the hot-exciton reservoir. Similarly, exciton-polariton interactions are a strong source of spin mixing [20] that, in the presence of an initial spin imbalance, result in changes of the relative spin populations that prevent the realisation of spinor polariton bistability. 


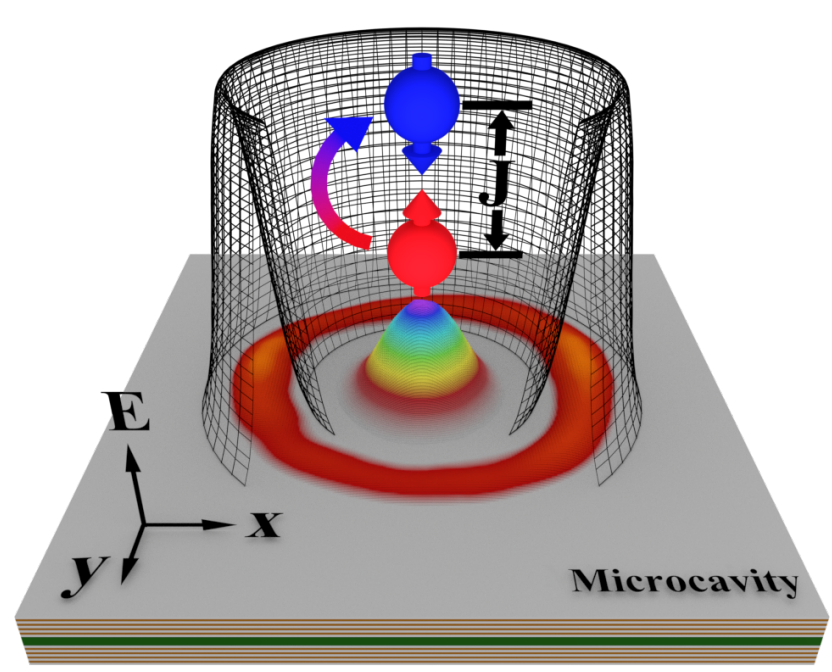

Figure 1. Schematic of the optical excitation scheme. The red ring on the surface of the microcavity is an intensity map of the pump beam, the black mesh annular barrier represents the confining potential due to the exciton reservoir. A section of the barrier is removed to show the trapped condensate shown as the rainbow-colored Gaussian mode in the center of the confining potential. The red and the blue spheres show the spin-up and spin-down states forming the condensate respectively. The arrow shows a current of polaritons between the two spin states.

In this letter, we realize spin-bistability under nonresonant optical pumping, in the absence of external fields, by spatially separating the spin-bistable polariton state from the hot-exciton reservoir, thus strongly suppressing spin depolarisation in the system. The spinbistability is evidenced on the spinor of a polariton condensate, trapped at the centre of an annular excitation beam, in the form of a hysteresis loop in the degree of circular polarization versus the optical excitation power. We attribute the spin-bistability to an interplay of polariton nonlinearities with the internal Josephson coupling of the two spin components. Figure 1 depicts a schematic of the system; the red ring on the surface of the microcavity, shows the pumping intensity profile. The rainbow colored Gaussian, in the centre of the ring, shows the measured polariton density of the ground state, $\psi_{00}$. The black mesh annular barrier represents the repulsive potential due to the optically injected hot-exciton reservoir. The red and blue spheres in the centre of the ring represent the two spin components of the polariton condensate that are coupled via an internal Josephson coupling, J.

The microcavity we use in this study is a $5 \lambda / 2$ cavity with GaAs quantum wells (QWs), as in [21], held at $\sim 6 \mathrm{~K}$ using a continuous flow cold finger cryostat. We excite the sample with a linearly polarized, continuous wave $(\mathrm{CW})$, monomode laser, tuned to the first reflectivity minimum at $754 \mathrm{~nm}$. A spatial light modulator (SLM) imprints a phase profile, and the desired annular pump
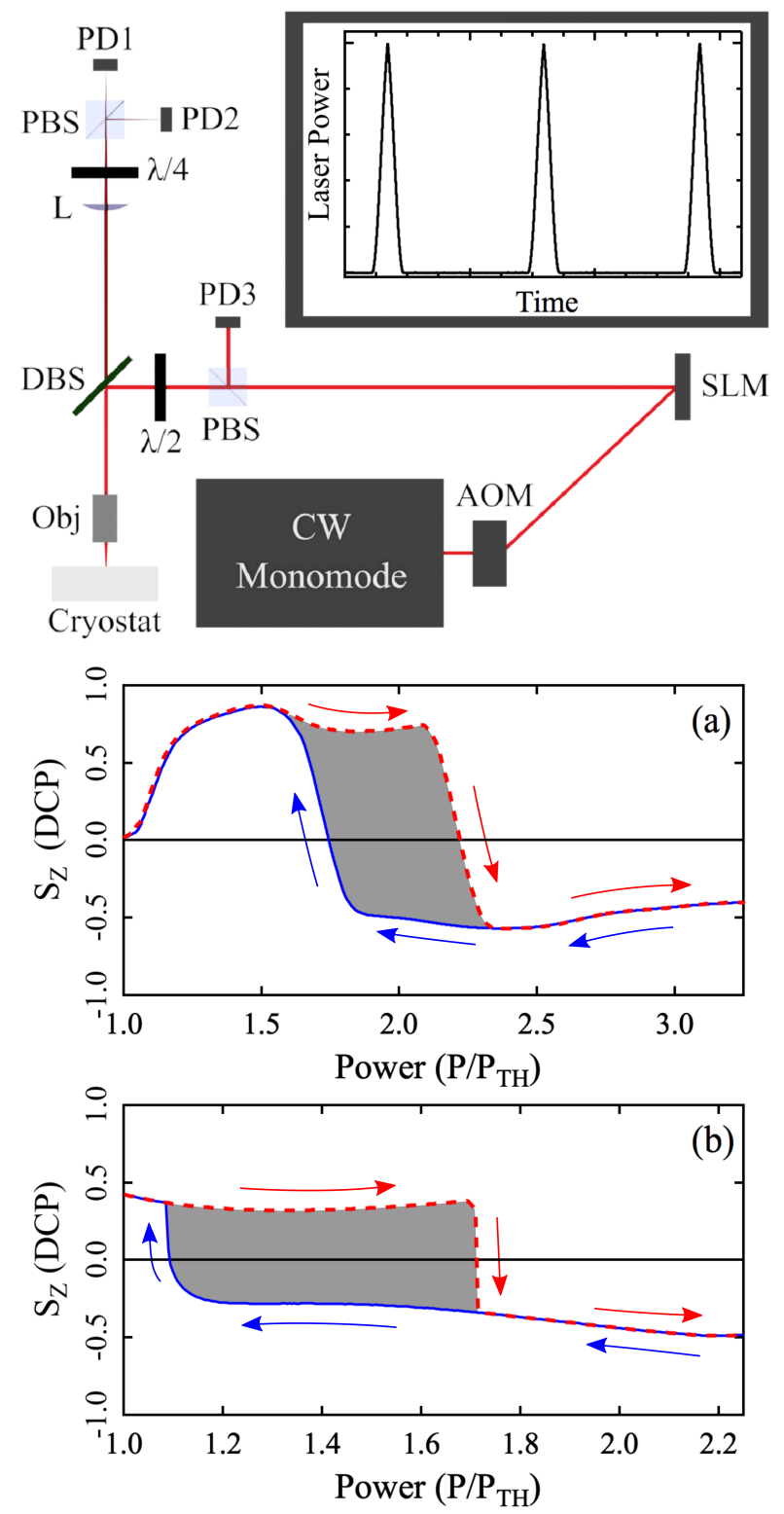

Figure 2. Schematic of the experimental setup; SLM - spatial light modulator, AOM - acousto-optical modulator, PBS -polarizing beam splitter, DBS - dichroic beam splitter, Obj microscope objective lens, L - plano-convex lens, $\lambda / 4$ - quater waveplate, $\lambda / 2$ - half waveplate and $\mathrm{PD}(1,2,3)$ photodiodes measuring the spin components of the photoluminescence and the corresponding laser intensity. (a) Measured third Stokes component, $S_{Z}$, representing the degree of circular polarization (DCP) vs pump power displaying the characteristic hysteresis loop of bistability for an annulus of $\sim 16.1 \mu \mathrm{m}$ diameter and $1.67 \mathrm{~ms}$ sweep time at a detuning of $-7.6 \mathrm{meV}$. (b) Hysteresis loop in $S_{Z}$ vs pump power resulting from the numerical simulations.

geometry is focused on the sample surface by a $0.4 \mathrm{nu}-$ merical aperture (NA) microscope objective lens. The intensity of the laser is modulated into triangular pulses using an acousto-optical modulator (AOM) that is driven by a triangular voltage pulse train. A polarization analyser is used to record the two cross-circular components 
of the photoluminescence intensity. The top schematic of Fig. 2 shows the setup and the triangular laser pulse train used to excite the sample. The use of a high NA excitation lens introduces a $\sim 10 \%$ ellipticity to the pump beam [22], which creates a small spin imbalance in the injected carriers. Since the electron spin relaxation time in GaAs QWs is longer than the carrier relaxation time to the exciton reservoir, the initial electron spin imbalance is reflected in the spin imbalance of the exciton reservoir that in turn leads to a spin polarized polariton condensate [23]; by separating the polariton condensate from the exciton reservoir here, we observe $93 \%$ degree of circular polarization (DCP).

Figure 2(a) shows a typical hysteresis loop in the DCP, represented by the $S_{Z}$ component of the Stokes polarization vector versus the pump power normalized to the condensation threshold at a detuning of $-7.6 \mathrm{meV}$. With increasing pump power, shown with a red dashed line in Fig. 2(a), we observe a spin reversal as it was previously observed in time integrated measurements [23]. The spin reversal was attributed to the transition into a desynchronization regime in Ref.[23] through the complex spin-dependent Ginsburg-Landau equations (GLE) with an internal Josephson coupling term between the two spinor components. With decreasing pump power (blue solid line), a backwards spin reversal is observed at a lower pumping power compared to the power for the spin reversal observed in the forward direction. The signature of spin bistability is qualitatively reproduced numerically with the same theoretical model of Ref.[23] as shown in Fig. 2(b); see Appendix A of the Supplemental Material.

To gain an understanding of the nature of polariton spin bistability, in the following we draw analogues with the driven damped pendulum. We non-dimensionalize the complex spin-dependent Ginsburg-Landau equation and the rate equation describing the density in the exciton-reservoir and reduce them to the equation of a driven-damped pendulum:

$$
\ddot{\Theta}+\beta(p) \dot{\Theta}=-I_{\text {bias }}(p)-I_{\text {cr }}(p) \sin \Theta,
$$

where $\Theta$ is the phase difference between two spin components, $\beta$ is a damping coefficient (positive above threshold) that increases with pumping power approaching a value of $1 / 2, I_{\text {bias }}$ is equivalent to the driving torque of a pendulum and $I_{\text {cr }}$ is equivalent to the maximum gravitational torque. A description of the process of non-dimensionalization and reduction is presented in Appendix A of the Supplemental Material.

A driven damped pendulum supports two types of solution [24]: if the torques' ratio $I=\left|I_{\text {bias }}\right| /\left|I_{\text {cr }}\right| \leq 1$, then the pendulum's trajectory is attracted to a fixed point (stationary solutions regime), where the angular displacement is constant $(\dot{\Theta}=0)$ for a fixed driving torque. Alternatively, if $I>1$, then $\dot{\Theta} \neq 0$, in which case the pendulum continues to overturn even under a fixed driving torque (limit cycle solutions regime). In this scheme, bistability occurs in the backward ramp of the driving torque due to its inertia, where the backward ramp corresponds to decreasing pump power.

Here, the stationary solutions regime results in the phases of the two spin states evolving synchronously with a constant phase difference; top branch of the bistability in Fig. 2(a,b). The limit cycle solutions regime, $\dot{\Theta} \neq 0$, results in the desynchronised evolution of the spin states' phases. With decreasing pump power, the role of the "inertia" increases due to the decrease of the damping rate $\beta$, which allows for the limit cycle solutions regime to persist for pump powers lower than those on the forward ramp, leading to the observed hysteresis. A schematic comparison between bistability in the driven damped pendulum and the spinor polariton condensate is presented in Appendix B of the Supplemental Material.

In the following, we investigate the dependence of the observed bistability on the overlap, $\Omega$, between the condensate and the hot-exciton reservoir. At low temperatures, the pump induced exciton reservoir is co-localised with the pump beam due to the small (sub-micron) exciton diffusion length, which allows us to control $\Omega$ by changing the diameter of the annular pump profile. For diameters ranging $11.8 \mu \mathrm{m}$ to $17.9 \mu \mathrm{m}$ polariton condensation occurs in a Gaussian-shaped ground state, $\psi_{00}$. Figure 3(a) shows the dependence of the hysteresis area on the diameter of the annular pump at a detuning of $-7.6 \mathrm{meV}$ (black diamonds). With increasing pump diameter from $11.8 \mu \mathrm{m}$ to $17.9 \mu \mathrm{m}$ the hysteresis area increases monotonically. The (red) dotted line in Fig.3(a) shows the calculated $\Omega$ assuming a Gaussian-shaped condensate and an annular pump profile with a Gaussian cross section, where the width of the condensate and the annular profile are approximated from the measured photoluminescence under different annular pump diameters. When the diameter of the annular pump exceeds $18 \mu \mathrm{m}$, the trapping potential allows for the coexistence of higher order Hermite-Gaussian-shaped, $\psi_{01}$, condensates with the $\psi_{00}$ condensate [25], this leads to the collapse of the hysteresis loop. The hysteresis loops vs the diameter of the annular pump are presented in Appendix $\mathrm{C}$ of the Supplemental Material. The deleterious effect of exciton-polariton interactions on bistability is further corroborated by changing the exciton fraction of polaritons. The solid black circle in Fig.3(a) shows the hysteresis area obtained for an annular pump diameter of $13.1 \mu \mathrm{m}$ at a detuning of $-11.2 \mathrm{meV}$.

Figure 3(b) shows the hysteresis area vs temperature ranging from $4 \mathrm{~K}$ to $40 \mathrm{~K}$, where strong coupling is sustained [26]. With increasing temperature the exciton linewidth, the exciton reservoir lifetime, the exciton diffusion length and the exciton-photon detuning are increasing. All of these effects contribute in the suppression of the hysteresis area. We note that the observed hysteresis loops under all previous excitation and detuning 

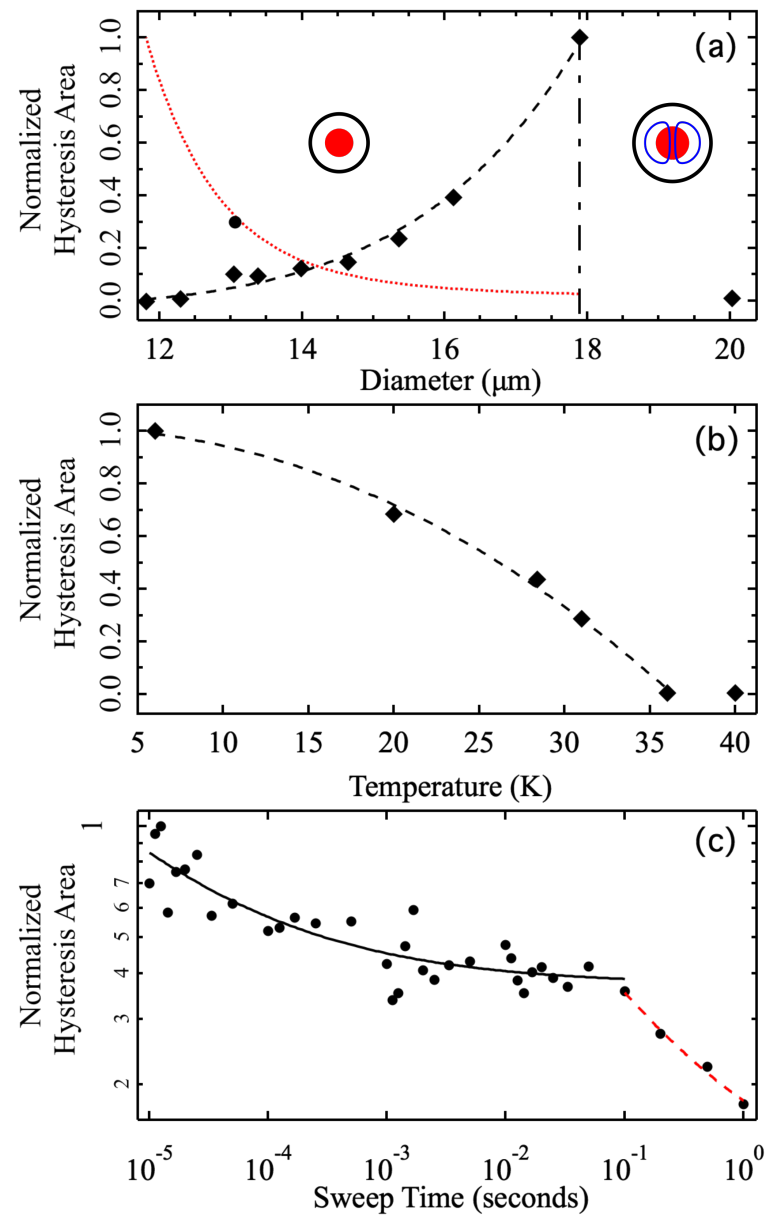

Figure 3. (a) The dependence of the hysteresis area (symbols) on the diameter of the annular pump at $\sim 6 K$. The dotted red line shows the estimated overlap between the condensate and exciton reservoir, $\Omega$, vs the annular pump diameter. The vertical dash-dotted line separates the regimes wherein only the ground state, $\psi_{00}$, or both $\psi_{00}$ and first excited state, $\psi_{01}$, coexists. (b) Temperature dependence of the hysteresis area for a $\sim 15.4 \mu \mathrm{m}$ diameter annular pump. The data in (a) and (b) are recorded with $1 \mathrm{~ms}$ sweep time. (c) Hysteresis area as a function of sweep time, $T_{P}$, for $\sim 13.1 \mu \mathrm{m}$ diameter annular pump recorded at $\sim 6 K$. The black (red) lines are power-law fits. In all of the above data points, solid circles are recorded at a detuning of $-11.2 \mathrm{meV}$, whilst solid diamonds at $-7.6 \mathrm{meV}$.

conditions disappears when we switch from mono-mode (low intensity fluctuations) to multi-mode CW excitation lasers; the latter induces density fluctuations in the exciton reservoir that are mirrored in the density of the condensate leading to the collapse of bistability [27].

Relevant to the applications in volatile memories and switching is the range of sweep times wherein the bistable behaviour is durable, i.e. non-collapsing hysteresis loops. To that end, we investigate the relationship of the hysteresis area on the duration of the sweep time $\left(T_{P}\right)$. Figure 3(c) shows the hysteresis area for sweep times spanning five orders of magnitude $T_{P} \in[10 \mu s, 1 s]$, where bistability is observed in the absence of collapses [28].
We observe that the dependence of the hysteresis area as a function of the sweep time exhibits a double power-law decay. The mean-field approach of the spin-dependent Ginzburg-Landau model implemented here to explain the observed bistability cannot reproduce the dependence of the hysteresis area on the sweep time. Recently, a double power-law decay vs the sweep time was attributed to quantum fluctuations [29]. An evolution from a double to a single power-law decay was experimentally observed with increasing the average photon number and was ascribed to a dissipative phase transition between the quantum regime and the thermodynamic limit [13]. The dissipative phase transition was shown to depend strongly on the laser-cavity detuning; here spinor bistability is observed under non-resonant optical pumping.

In summary, we demonstrate optical bistability in spinor polariton condensates under non-resonant optical excitation in the absence of external biasing fields. The hysteresis loop can be qualitatively modelled through the complex spin-dependent Ginsburg-Landau equations with an internal Josephson coupling term between the two spinor components. We unravel the detrimental role of a hot-exciton reservoir on bistability by controlling the overlap of the pump induced exciton reservoir and the polariton condensate. The dependence of spin bistability on the condensate's overlap with the exciton reservoir allows us to control the hysteresis area by tuning the excitation geometry. Most importantly, we obtain noncollapsing bistability hysteresis loops for a record range of sweep times, $[10 \mu s, 1 s]$, offering a promising route to spin switches and spin memory elements.

\section{ACKNOWLEDGEMENTS}

This work was carried out in the framework of the joint Russian-Greek project supported by Ministry of Education and Science of The Russian Federation (project RFMEFI61617X0085), the Skoltech NGP Program (Skoltech-MIT joint project), and the UK's Engineering and Physical Sciences Research Council (grant EP/M025330/1 on Hybrid Polaritonics).

[1] Abraham, E. and Smith, S. D. Optical bistability and related devices Reports on Progress in Physics 45815 (1982)

[2] Gibbs, Hyatt Optical Bistability: Controlling Light With Light (Elsevier, 2012)

[3] Joshi, Amitabh and Brown, Andy and Wang, Hai and Xiao, Min. Controlling optical bistability in a three-level atomic system Physical Review A 67041801 (2003)

[4] Jung, Peter and Gray, George and Roy, Rajarshi and Mandel, Paul. Scaling law for dynamical hysteresis Physical Review Letters 651873 (1990)

[5] Forsmann, F. and Jäger, D. and Niessen, W. Nonresonant optical bistability in InP:Fe seed devices. Optics Communications 62193 (1987)

[6] Smith, PW and Turner, EH. A bistable Fabry-Perot resonator. Applied Physics Letters 30280 (1977) 
[7] Gibbs, HM and McCall, SL and Venkatesan, TNC. Differential gain and bistability using a sodium-filled FabryPerot interferometer. Physical Review Letters 361135 (1976)

[8] Paraïso, T. K. and Wouters, M. and Léger, Y. and Morier-Genoud, F. and Deveaud-Plédran, B. Multistability of a coherent spin ensemble in a semiconductor microcavity Nature Materials 9655 (2010)

[9] Baas, A. and Karr, J. Ph. and Eleuch, H. and Giacobino, E. Optical bistability in semiconductor microcavities Physical Review A 69023809 (2004)

[10] Baas, A. and Karr, J.-Ph. and Romanelli, M. and Bramati, A. and Giacobino, E. Optical bistability in semiconductor microcavities in the nondegenerate parametric oscillation regime: Analogy with the optical parametric oscillator Physical Review B 70161307 (2004)

[11] Cerna, R. and Léger, Y. and Paraïso, T. K. and Wouters, M. and Morier-Genoud, F. and Portella-Oberli, M. T. and Deveaud, B. Ultrafast tristable spin memory of a coherent polariton gas. Nature Communications 42008 (2013)

[12] Ouellet-Plamondon, C. and Sallen, G. and MorierGenoud, F. and Oberli, D. Y. and Portella-Oberli, M. T. and Deveaud, B. Reservoir-induced decoherence of resonantly excited confined polaritons. Physical Review B 95 085302 (2017)

[13] Rodriguez, S. R. K. and Casteels, W. and Storme, F. and Carlon Zambon, N. and Sagnes, I. and Le Gratiet, L. and Galopin, E. and Lemaître, A. and Amo, A. and Ciuti, C. and Bloch, J. Probing a Dissipative Phase Transition via Dynamical Optical Hysteresis Physical Review Letters 118247402 (2017)

[14] Dreismann, Alexander and Ohadi, Hamid and del ValleInclan Redondo, Yago and Balili, Ryan and Rubo, Yuri G. and Tsintzos, Simeon I. and Deligeorgis, George and Hatzopoulos, Zacharias and Savvidis, Pavlos G. and Baumberg, Jeremy J. A sub-femtojoule electrical spinswitch based on optically trapped polariton condensates Nature Materials 151074 (2016)

[15] Bajoni, Daniele and Semenova, Elizaveta and Lemaître, Aristide and Bouchoule, Sophie and Wertz, Esther and Senellart, Pascale and Barbay, Sylvain and Kuszelewicz, Robert and Bloch, Jacqueline. Optical Bistability in a GaAs-Based Polariton Diode. American Physical Society 101266402 (2008)

[16] Amthor, M. and Liew, T. C. H. and Metzger, C. and Brodbeck, S. and Worschech, L. and Kamp, M. and Shelykh, I. A. and Kavokin, A. V. and Schneider, C. and Höfling, S. Optical bistability in electrically driven polariton condensates. Physical Review B 91081404 (2015)

[17] Kyriienko, O. and Ostrovskaya, E. A. and Egorov, O. A. and Shelykh, I. A. and Liew, T. C. H. Bistability in microcavities with incoherent optical or electrical excitation Physical Review B 90125407 (2014)

[18] Askitopoulos, A. and Ohadi, H. and Kavokin, A. V. and Hatzopoulos, Z. and Savvidis, P. G. and Lagoudakis,
P. G. Polariton condensation in an optically induced two-dimensional potential Physical Review B $8 \mathbf{8} 041308$ (2013)

[19] Krizhanovskii, D. N. and Lagoudakis, K. G. and Wouters, M. and Pietka, B. and Bradley, R. A. and Guda, K. and Whittaker, D. M. and Skolnick, M. S. and DeveaudPlédran, B. and Richard, M. and André, R. and Dang, Le Si. Coexisting nonequilibrium condensates with longrange spatial coherence in semiconductor microcavities. Physical Review B 80045317 (2009)

[20] Kavokin, A. and Lagoudakis, P. G. and Malpuech, G. and Baumberg, J. J. Polarization rotation in parametric scattering of polaritons in semiconductor microcavities. Physical Review B 67195321 (2003)

[21] Kammann, E. and Liew, T. C. H. and Ohadi, H. and Cilibrizzi, P. and Tsotsis, P. and Hatzopoulos, Z. and Savvidis, P. G. and Kavokin, A. V. and Lagoudakis, P. G. Nonlinear Optical Spin Hall Effect and Long-Range Spin Transport in Polariton Lasers. Physical Review Letters 109036404 (2012)

[22] Cilibrizzi, P. and Sigurdsson, H. and Liew, T. C. H. and Ohadi, H. and Wilkinson, S. and Askitopoulos, A. and Shelykh, I. A. and Lagoudakis, P. G. Polariton spin whirls Physical Review B 92155308 (2015)

[23] Askitopoulos, A. and Kalinin, K. and Liew, T. C. H. and Cilibrizzi, P. and Hatzopoulos, Z. and Savvidis, P. G. and Berloff, N. G. and Lagoudakis, P. G. Nonresonant optical control of a spinor polariton condensate Physical Review B 93205307 (2016)

[24] Strogatz, Steven H. Nonlinear Dynamics and Chaos: With Applications to Physics, Biology, Chemistry and Engineering. Perseus Books (2000)

[25] Askitopoulos, A. and Liew, T. C. H. and Ohadi, H. and Hatzopoulos, Z. and Savvidis, P. G. and Lagoudakis, P. G. Robust platform for engineering pure-quantum-state transitions in polariton condensates Physical Review B 92035305 (2015)

[26] Tsotsis, P. and Eldridge, P. S. and Gao, T. and Tsintzos, S. I. and Hatzopoulos, Z. and Savvidis, P. G. Lasing threshold doubling at the crossover from strong to weak coupling regime in GaAs microcavity New Journal of Physics 14023060 (2012)

[27] Abbaspour, H. and Sallen, G. and Trebaol, S. and Morier-Genoud, F. and Portella-Oberli, M. T. and Deveaud, B. Effect of a noisy driving field on a bistable polariton system Physical Review B 92165303 (2015)

[28] For all the measurements here, the ratio of the time interval between successive sweeps, $T_{S e p}$, and the sweep time, $T_{P}$, is kept constant at $T_{S e p} / T_{P}=5$.

[29] Casteels, W. and Storme, F. and Le Boité, A. and Ciuti, C. Power laws in the dynamic hysteresis of quantum nonlinear photonic resonators Physical Review A 93033824 (2016) 